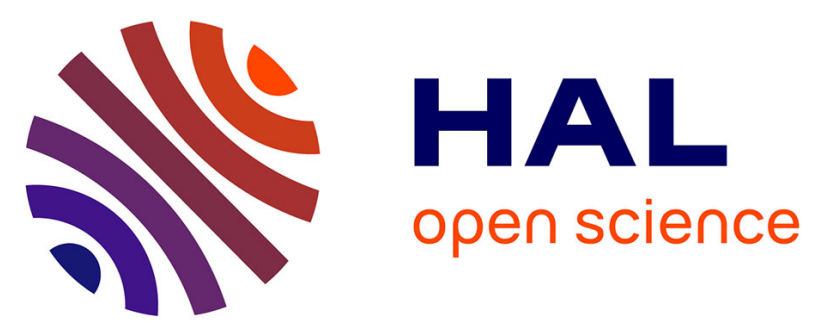

\title{
Explicit Complementary Observer Design on Special Linear Group SL(3) for Homography Estimation using Conic Correspondences
}

\author{
Minh-Duc Hua, Tarek Hamel, Robert Mahony, Guillaume Allibert
}

\section{To cite this version:}

Minh-Duc Hua, Tarek Hamel, Robert Mahony, Guillaume Allibert. Explicit Complementary Observer Design on Special Linear Group SL(3) for Homography Estimation using Conic Correspondences. 56th IEEE Conference on Decision and Control (CDC), Dec 2017, Melbourne, Australia. hal-01628177

\section{HAL Id: hal-01628177 \\ https://hal.science/hal-01628177}

Submitted on 2 Nov 2017

HAL is a multi-disciplinary open access archive for the deposit and dissemination of scientific research documents, whether they are published or not. The documents may come from teaching and research institutions in France or abroad, or from public or private research centers.
L'archive ouverte pluridisciplinaire HAL, est destinée au dépôt et à la diffusion de documents scientifiques de niveau recherche, publiés ou non, émanant des établissements d'enseignement et de recherche français ou étrangers, des laboratoires publics ou privés. 


\title{
Explicit Complementary Observer Design on Special Linear Group $S L(3)$ for Homography Estimation using Conic Correspondences
}

\author{
Minh-Duc Hua, Tarek Hamel, Robert Mahony, Guillaume Allibert
}

\begin{abstract}
This paper presents a new observer on the Special Linear group $S L(3)$. The proposed solution is oriented towards online homography estimation from video sequences obtained from robotic vehicles equipped with a monocular camera. The originality of this paper relies on the extension of our prior works based on linear features to nonlinear features and more particularly to conics. A comprehensive observability analysis is provided. Performance and robustness of the proposed observer are evaluated in both simulations and experiments.
\end{abstract}

\section{INTRODUCTION}

The homography is an invertible mapping relating two views of the same planar scene. It plays a key role in many computer vision and robotics applications, especially those involving man-made environments composed of planar surfaces. The problem of homography estimation has been extensively studied (e.g. [2], [5]). However, most existing works only consider the homography as an incidental variable and are not focused on improving the estimation over time. The quality of the homography estimates depends heavily on the nature of the image features exploited as well as the algorithm used. Image features for homography estimation are typically geometric (points, lines, conics, etc.) or texture. For a well textured scene, state-of-the-art methods can provide high quality homography estimates at the cost of high computational complexity (see [5]). For a scene with poor texture, while significant computational effort is still required, the poor quality of homography estimates is often an important issue. This is particularly the case in varying lighting conditions and in presence of specular reflections or moving shadows, where photogrammetric error criteria used in texture-based methods become ill-posed. Featurebased methods of image-to-image homography estimation are robust to these issues as long as good features and good matching are obtained. They have been the mainstay of robust computer vision in the past and most well-known algorithms rely on point or line correspondences [2], [5]. Homography estimation based on nonlinear features such as conics or contours has been less explored [7], [8]. As opposed to linear images features, conics encode richer geometric information so that two conic correspondences may be sufficient to compute the image-to-image homography whereas at least 4 point (or 4 line) correspondences are

M.-D. Hua, T. Hamel and G. Allibert are with I3S, University Côte d'Azur, CNRS, Sophia Antipolis, France. email: hua (thamel, allibert) @i3s.unice.fr.

R. Mahony is with the Research School of Engineering, Australian National University, Canberra, Australia. email: Robert.Mahonyeanu.edu.au. required for the same task [5]. Based on at least 2 conic correspondences, existing approaches compute homographies from linear algebraic equations [3], [9], [15] or polynomial equations [8], and by solving eigenvalue/eigenvector problem [3] or eigen-decomposition problem [9]. Most of them, however, restrict the type of conics to ellipses.

One of the objectives of the present work is to develop a feature-based homography estimation algorithm that exploits the temporal correlation of data across a video sequence rather than computing algebraically individual raw homography for each image. The methodology taken exploits the underlying structure of the Special Linear group $S L(3)$, a Lie group isomorphic to the group of homographies. This property has, however, seldom been considered in observer design for smoothing the homography estimates [11], [13]. The proposed approach is the continuation of our prior works on the formulation of point (resp. line) feature-based innovation that incorporates directly point (resp. line) correspondences in the observer without requiring the prior reconstruction of individual homographies [4] (resp. [6]). The features here exploited are conics, which are of higher-level than point and line features. The originality of this work relies on the capacity of the observer of encompassing static and nonstatic cases, temporal smoothing of noisy data, and providing good homography estimates even in situations where the number and quality of conic-feature correspondences on a frame-by-frame basis are insufficient to directly compute raw homographies, during a short time period. Beyond the technical contributions, this work contributes to our ongoing development of a unified observer on $S L(3)$ that offers large convergence domain and low computational complexity and that can exploit all types of features, either geometric or texture, for homography estimation.

The paper is organized as follows. Section II recalls some algebraic equations of homographies and conics. Section III is devoted to observer design and observability analysis. The observer is derived from the theory of "invariant observers based on homogeneous output space" [12] by adequately representing the conic measurement in the homogeneous space. A comprehensible observability analysis is also provided, offering a generalization of existing studies on this topic. In Section IV simulation results involving different types of conics and large initial errors are presented to illustrate the observer performance. Experimental results are reported in Section V, showing that real-time implementation is possible and that the observer performance is still acceptable in situations where the observability is temporarily lost. Finally, a short concluding section follows. 


\section{TECHNICAL BACKGROUND}

A. Notation

- The Special Linear group $S L(3)$ and its algebra $\mathfrak{s l}(3)$ are

$$
\begin{aligned}
& S L(3) \triangleq\left\{\mathbf{H} \in \mathbb{R}^{3 \times 3} \mid \operatorname{det} \mathbf{H}=1\right\}, \\
& \mathfrak{s l}(3) \triangleq\left\{\mathbf{U} \in \mathbb{R}^{3 \times 3} \mid \operatorname{tr}(\mathbf{U})=0\right\} .
\end{aligned}
$$

- The adjoint operator is a mapping Ad : $S L(3) \times \mathfrak{s l}(3) \rightarrow$ $\mathfrak{s l}(3)$ defined by

$$
\operatorname{Ad}_{\mathbf{H}} \mathbf{U} \triangleq \mathbf{H U H}^{-1}, \mathbf{H} \in S L(3), \mathbf{U} \in \mathfrak{s l}(3) .
$$

- The projection operator $\mathbb{P}: \mathbb{R}^{3 \times 3} \rightarrow \mathfrak{s l}(3)$ is defined by

$$
\mathbb{P}(\mathbf{A}) \triangleq \mathbf{A}-\frac{1}{3} \operatorname{tr}(\mathbf{A}) \mathbf{I},
$$

with I the identity matrix.

- The Frobenius norm is defined by $\|\mathbf{A}\|_{F} \triangleq \sqrt{\operatorname{tr}\left(\mathbf{A} \mathbf{A}^{\top}\right)}$, $\forall \mathbf{A} \in \mathbb{R}^{3 \times 3}$. For any symmetric positive definite matrix $\mathbf{K} \in$ $\mathbb{R}^{3 \times 3}$, the norm $\|\mathbf{A}\|_{\mathbf{K}}, \forall \mathbf{A} \in \mathbb{R}^{3 \times 3}$, is defined by $\|\mathbf{A}\|_{\mathbf{K}} \triangleq$ $\left\|\mathbf{A} \mathbf{K}^{\frac{1}{2}}\right\|_{F}=\sqrt{\operatorname{tr}\left(\mathbf{A K} \mathbf{A}^{\top}\right)}$.

- Let $\langle\cdot, \cdot\rangle: \mathfrak{s l}(3) \times \mathfrak{s l}(3) \longrightarrow \mathbb{R}$ be an inner product on $\mathfrak{s l}(3)$, chosen to be the Euclidean matrix inner product on $\mathbb{R}^{3 \times 3}$. Then, a right-invariant Riemannian metric on $\operatorname{SL}(3)$ induced by the inner product $\langle\cdot, \cdot\rangle$ is defined by

$\left\langle\mathbf{U}_{1} \mathbf{H}, \mathbf{U}_{2} \mathbf{H}\right\rangle_{\mathbf{H}} \triangleq\left\langle\mathbf{U}_{1}, \mathbf{U}_{2}\right\rangle, \mathbf{H} \in S L(3), \mathbf{U}_{1}, \mathbf{U}_{2} \in \mathfrak{s l}(3)$.

- $\operatorname{grad}_{1} f(\cdot, \cdot)$ and $\operatorname{Hess}_{1} f(\cdot, \cdot)$ denote the gradient and Hessian in the first variable of $f(\cdot, \cdot)$, respectively.

\section{B. Homographies}

Let $\mathcal{A}$ (resp. $\mathcal{A}$ ) denote projective coordinates for the image plane of a camera $\AA$ (resp. $A$ ), and let $\{\AA\}$ (resp. $\{A\})$ denote its reference (resp. current) frame. Let $\xi \in \mathbb{R}^{3}$ denote the position of the frame $\{A\}$ with respect to $\{\AA$ 의 expressed in $\{\AA\}$. The orientation of the frame $\{A\}$ with respect to $\{\AA$ \} $\}$, is given by a rotation matrix $\mathbf{R} \in S O(3)$, with $\mathbf{R}:\{A\} \rightarrow\{\AA$ \} $\}$ as a mapping. In addition, we denote by $\stackrel{\circ}{d}$ (resp. $d$ ) and $\dot{\eta}$ (resp. $\eta$ ) respectively the distance from the origin of $\{\AA\}$ (resp. $\{A\}$ ) to the planar scene and the normal to the scene expressed in $\{\AA$ \} (resp. $\{A\}$ ). One has

$$
\stackrel{\circ}{\mathbf{P}}=\mathbf{R P}+\boldsymbol{\xi}
$$

as a relation between the coordinates of the same point in the reference frame $(\stackrel{\circ}{\mathbf{P}} \in\{\stackrel{\circ}{A}\})$ and in the current frame $(\mathbf{P} \in$ $\{A\})$. The camera internal parameters, in the commonly used approximation [5], define a calibration matrix $\mathbf{K} \in \mathbb{R}^{3 \times 3}$ so that ${ }^{1}$ :

$$
\stackrel{\circ}{\mathbf{p}} \cong \mathbf{K} \stackrel{\circ}{\mathbf{P}}, \quad \mathbf{p} \cong \mathbf{K P},
$$

where $\mathbf{p} \in \mathcal{A}$ is the image of a point when the camera is aligned with frame $\{A\}$, and can be written as $(u, v, 1)^{\top}$ using the homogeneous coordinate representation for that $2 \mathrm{D}$ image point. Likewise, $\stackrel{\circ}{\mathbf{p}} \in \mathcal{A}$ is the image of the same point viewed when the camera is aligned with frame $\{\AA$.

The image homography matrix that maps pixel coordinates from $\mathcal{A}$ to $\mathcal{A}$ is given by

$$
\mathbf{H}_{\mathrm{im}}=\gamma \mathbf{K}\left(\mathbf{R}+\frac{\boldsymbol{\xi} \boldsymbol{\eta}^{\top}}{d}\right) \mathbf{K}^{-1}
$$

with $\gamma$ a scale factor. Without loss of generality, $\gamma$ can be chosen so that $\mathbf{H}_{\mathrm{im}} \in S L(3)$. In this case, $\gamma$ is equal $(d / \stackrel{\circ}{d})^{\frac{1}{3}}$

\footnotetext{
${ }^{1}$ Most statements in projective geometry involve equality up to a multiplicative constant denoted by $\cong$.
}

and corresponds to the second singular value of $\mathbf{H}_{\mathrm{im}}$ [10]. The Euclidean homography $\mathbf{H} \in S L(3)$ is related to $\mathbf{H}_{\mathrm{im}}$ by

$$
\mathbf{H}=\mathbf{K}^{-1} \mathbf{H}_{\mathrm{im}} \mathbf{K}=\gamma\left(\mathbf{R}+\frac{\boldsymbol{\xi} \boldsymbol{\eta}^{\top}}{d}\right) .
$$

We have $\mathbf{P} \cong \mathbf{H}^{-1} \stackrel{\circ}{\mathbf{P}}$ and $\mathbf{p} \cong \mathbf{H}_{\mathrm{im}}^{-1} \stackrel{\circ}{\mathbf{p}}$.

\section{Conics and conic correspondences}

In the Cartesian coordinate system, the general equation of a conic is of the form

$$
a x^{2}+2 b x y+c y^{2}+2 d x z+2 e y z+f z^{2}=0,
$$

with all coefficients real numbers and $a, b, c$ not all null, which in the homogeneous form can be written as

$$
\mathbf{P}^{\top} \mathbf{C P}=0
$$

with $\mathbf{C} \in \mathbb{R}^{3 \times 3}$ the conic in the homogeneous form

$$
\mathbf{C}=\left[\begin{array}{lll}
a & b & d \\
b & c & e \\
d & e & f
\end{array}\right]
$$

In this paper only non-degenerate conics are considered so that their determinant is not null. Since the relation $\mathbf{P}^{\top} \mathbf{C P}=$ 0 implies that $\mathbf{P}^{\top}(\lambda \mathbf{C}) \mathbf{P}=0$ for any number $\lambda \neq 0$, all $\lambda \mathbf{C}$ represent the same conic. Therefore, without loss of generality it can be assumed that $\mathbf{C}$ belongs to the set $\mathbb{C}$ defined as

$$
\mathbb{C} \triangleq\left\{\mathbf{C} \in \mathbb{R}^{3 \times 3} \mid \mathbf{C}^{\top}=\mathbf{C}, \operatorname{det}(\mathbf{C})=1\right\} .
$$

If $\mathbf{C}$ is non-degenerate, its determinant $\Delta=b^{2}-a c$ defines the type of conic.

- If $\Delta<0, \mathbf{C}$ is an ellipse;

- if $\Delta>0, \mathbf{C}$ is a hyperbola;

- if $\Delta=0, \mathbf{C}$ is a parabola.

From two views, the conic correspondence $\stackrel{\circ}{\mathbf{C}} \in\{\stackrel{\circ}{A}\}$ and $\mathbf{C} \in\{A\}$ of the same conic is related by a homography as

$$
\mathbf{C}=\mathbf{H}^{\top} \stackrel{\text { C }}{\mathbf{H}} \text {. }
$$

In fact, for any point belonging to the conic one has $\mathbf{P}^{\top} \mathbf{C P}=\stackrel{\circ}{\mathbf{P}}^{\top} \dot{\mathbf{C}} \dot{\mathbf{P}}=0$. Then, replacing the relation $\stackrel{\circ}{\mathbf{P}} \cong$ $\mathbf{H P}$ into $\stackrel{\circ}{\mathbf{P}}^{\top} \stackrel{\circ}{\mathbf{C}} \stackrel{\circ}{\mathbf{P}}=0$ one obtains $\mathbf{P}^{\top}\left(\mathbf{H}^{\top} \stackrel{\mathbf{C}}{\mathbf{H}}\right) \mathbf{P}=0$, thus yielding (2) using the fact that $\operatorname{det}(\mathbf{C})=\operatorname{det}(\dot{\mathbf{C}})=$ $\operatorname{det}(\mathbf{H})=1$.

The Euclidean conic $\mathbf{C} \in \mathbb{C}$ and its associated image conic $\mathbf{C}_{\mathrm{im}} \in \mathbb{C}$ are related by $\mathbf{C} \cong \mathbf{K}^{\top} \mathbf{C}_{\mathrm{im}} \mathbf{K}$.

\section{NONLINEAR OBSERVER DESIGN ON SL(3) BASED ON COPLANAR CONIC CORRESPONDENCES}

In this section an observer for $\mathbf{H} \in S L(3)$ is developed from a recent advanced theory for nonlinear observer design directly on the output space [12]. 


\section{A. Kinematics and measurements}

Consider the kinematics of $S L(3)$ given by

$$
\dot{\mathbf{H}}=F(\mathbf{H}, \mathbf{U}) \triangleq \mathbf{H U},
$$

with $\mathbf{U} \in \mathfrak{s l}(3)$ the group velocity. Assume that $\mathbf{U}$ is measured. Moreover, we consider a set of $N(\geq 2)$ conic measurements $\mathbf{C}_{k} \in \mathbb{C}$. All measurements are expressed in the camera current frame:

$$
\mathbf{C}_{k}=h\left(\mathbf{H}, \stackrel{\mathbf{C}}{k}_{k}\right) \triangleq \mathbf{H}^{\top} \stackrel{\circ}{\mathbf{C}}_{k} \mathbf{H}, k=\{1, \ldots, N\},
$$

where $\stackrel{\circ}{\mathbf{C}}_{k} \in \mathbb{C}$ are constant and known. For later use, define

$$
\stackrel{\mathrm{C}}{\triangleq}\left(\stackrel{\mathbf{C}}{1}_{1}, \cdots, \stackrel{\circ}{\mathbf{C}}_{N}\right), \quad \mathbf{C} \triangleq\left(\mathbf{C}_{1}, \cdots, \mathbf{C}_{N}\right) .
$$

One verifies that $S L(3)$ is a symmetry group with group actions

$$
\begin{aligned}
& \phi(\mathbf{Q}, \mathbf{H}) \triangleq \mathbf{H Q}, \\
& \psi(\mathbf{Q}, \mathbf{U}) \triangleq \operatorname{Ad}_{\mathbf{Q}^{-1}} \mathbf{U}=\mathbf{Q}^{-1} \mathbf{U} \mathbf{Q}, \\
& \rho\left(\mathbf{Q}, \stackrel{\mathbf{C}}{k}_{k}\right) \triangleq \mathbf{Q}^{\top} \mathbf{C}_{k} \mathbf{Q},
\end{aligned}
$$

with $\mathbf{Q} \in S L(3)$ and $\mathbf{U} \in \mathfrak{s l}(3)$. They are right group actions in the sense of [12]. The kinematics are right equivariant since it is verified that

$$
\begin{aligned}
& \rho\left(\mathbf{Q}, h\left(\mathbf{H}, \stackrel{\mathbf{C}}{k}_{k}\right)\right)=h\left(\phi(\mathbf{Q}, \mathbf{H}), \stackrel{\mathbf{C}}{k}_{k}\right), \\
& d \phi_{\mathbf{Q}}(\mathbf{H})[F(\mathbf{H}, \mathbf{U})]=F(\phi(\mathbf{Q}, \mathbf{H}), \psi(\mathbf{Q}, \mathbf{U})) .
\end{aligned}
$$

B. Observer design on $S L(3)$ based on conic correspondences

Let $\hat{\mathbf{H}} \in S L(3)$ denote the estimate of $\mathbf{H}$. Define the right group error $\mathbf{E}:=\hat{\mathbf{H}} \mathbf{H}^{-1} \in S L(3)$ so that $\hat{\mathbf{H}}$ converges to $\mathbf{H}$ if and only if $\mathbf{E}$ converges to identity. Define the output errors $\mathbf{e}_{k} \in \mathbb{C}$, with $k \in\{1, \cdots, N\}$ as:

$$
\mathbf{e}_{k} \triangleq \rho\left(\hat{\mathbf{H}}^{-1}, \mathbf{C}_{k}\right)=\hat{\mathbf{H}}^{-\top} \mathbf{C}_{k} \hat{\mathbf{H}}^{-1}=\mathbf{E}^{-\top} \stackrel{\mathbf{C}}{k}_{k} \mathbf{E}^{-1} .
$$

The proposed observer takes the following general form

$$
\dot{\hat{\mathbf{H}}}=\hat{\mathbf{H}} \mathbf{U}-\boldsymbol{\Delta}(\hat{\mathbf{H}}, \mathbf{C}) \hat{\mathbf{H}}
$$

where $\boldsymbol{\Delta}(\hat{\mathbf{H}}, \mathbf{C}) \in \mathfrak{s l}(3)$ is the innovation term to be designed thereafter and must be right equivariant in the sense that

$\boldsymbol{\Delta}\left(\phi(\mathbf{Q}, \hat{\mathbf{H}}), \rho\left(\mathbf{Q}, \mathbf{C}_{1}\right), \ldots, \rho\left(\mathbf{Q}, \mathbf{C}_{N}\right)\right)=\boldsymbol{\Delta}\left(\hat{\mathbf{H}}, \mathbf{C}_{1}, \ldots, \mathbf{C}_{N}\right)$ for all $\mathbf{Q} \in S L(3)$. Interestingly, if $\boldsymbol{\Delta}$ is right equivariant, the dynamics of $\mathbf{E}$ are autonomous and given by [12, Th. 1]:

$$
\dot{\mathbf{E}}=-\Delta(\mathbf{E}, \stackrel{\mathbf{C}}{)} \mathbf{E}
$$

To determine $\boldsymbol{\Delta}(\hat{\mathbf{H}}, \mathbf{C})$, a non-degenerate right-invariant cost function is needed. To this purpose, we first define individual degenerate right-invariant costs at $\dot{\mathbf{C}}_{k}$ on the output spaces as follows:

$$
\mathcal{C}_{\dot{\mathbf{C}}_{k}}\left(\hat{\mathbf{H}}, \mathbf{C}_{k}\right) \triangleq \frac{1}{2}\left\|\hat{\mathbf{H}}^{-\top} \mathbf{C}_{k} \hat{\mathbf{H}}^{-1}-\stackrel{\circ}{\mathbf{C}}_{k}\right\|_{\mathbf{K}_{k}}^{2},
$$

with $\mathbf{K}_{k} \in \mathbb{R}^{3 \times 3}$ symmetric positive definite matrix. One verifies that $\mathcal{C}_{\mathbf{C}_{k}}\left(\hat{\mathbf{H}}, \mathbf{C}_{k}\right)$ are right-invariant in the sense that $\mathcal{C}_{\dot{\mathbf{C}}_{k}}\left(\phi(\mathbf{Q}, \hat{\mathbf{H}}), \rho\left(\mathbf{Q}, \mathbf{C}_{k}\right)\right)=\mathcal{C}_{\dot{\mathbf{C}}_{k}}\left(\hat{\mathbf{H}}, \mathbf{C}_{k}\right), \forall \mathbf{Q} \in S L(3)$. Then, the aggregate cost defined as the sum of all the individual costs

$$
\mathcal{C}(\hat{\mathbf{H}}, \mathbf{C}) \triangleq \sum_{k=1}^{N} \mathcal{C}_{\mathbf{C}_{k}}\left(\hat{\mathbf{H}}, \mathbf{C}_{k}\right)
$$

is also right-invariant. According to [12, Lem. 3], the aggregate cost is non-degenerate if

$$
\bigcap_{k=1}^{N} \operatorname{stab}_{\rho}\left(\stackrel{\mathbf{C}}{k}_{k}\right)=\{\mathbf{I}\}
$$

where the stabilizer $\operatorname{stab}_{\rho}\left(\stackrel{\circ}{\mathbf{C}}_{k}\right)$ of an element $\stackrel{\circ}{\mathbf{C}}_{k} \in \mathbb{C}$ is defined by

$$
\operatorname{stab}_{\rho}\left(\stackrel{\circ}{\mathbf{C}}_{k}\right) \triangleq\left\{\mathbf{Q} \in S L(3) \mid \rho\left(\mathbf{Q}, \stackrel{\circ}{\mathbf{C}}_{k}\right)=\stackrel{\circ}{\mathbf{C}}_{k}\right\} .
$$

In fact, (9) is equivalent to

$$
\bigcap_{k=1}^{N} \operatorname{ker}\left(\mathrm{d} \rho_{\dot{\mathbf{C}}_{k}}(\mathbf{I})\right)=\{\mathbf{0}\},
$$

with $\operatorname{ker}\left(\mathrm{d} \rho_{\dot{\mathbf{C}}_{k}}(\mathbf{I})\right)$ the Lie-algebra associated with $\operatorname{stab}_{\rho}\left(\stackrel{\circ}{\mathbf{C}}_{k}\right)$.

Lemma 1 (See [12, Lem. 3]) If (10) is satisfied, then the aggregate cost $\mathcal{C}(\hat{\mathbf{H}}, \mathbf{C})$ defined by (8) is non-degenerate and $(\mathbf{I}, \mathbf{C})$ is a global minimum of $\mathcal{C}(\hat{\mathbf{H}}, \mathbf{C})$.

In fact, condition (10) ensures that the Hessian $\operatorname{Hess}_{1} \mathcal{C}(\mathbf{I}, \mathbf{C})$ is positive definite [12, Lem. 3]. In Section III$\mathrm{C}$, more explicit sufficient conditions will be provided and interpreted so that (10) is satisfied. For instance, assume that Lemma 1 is valid, the innovation term $\boldsymbol{\Delta}(\hat{\mathbf{H}}, \mathbf{C})$ involved in (6) is computed as [12, Eq. (40)]

$$
\boldsymbol{\Delta}(\hat{\mathbf{H}}, \mathbf{C})=\left(\operatorname{grad}_{1} \mathcal{C}(\hat{\mathbf{H}}, \mathbf{C})\right) \hat{\mathbf{H}}^{-1},
$$

using a right-invariant Riemannian metric on $S L(3)$. As a direct result of [12], the innovation term $\boldsymbol{\Delta}(\hat{\mathbf{H}}, \mathbf{C})$ defined by (11) is right-equivariant. Using standard rules for transformations of Riemannian gradients and the fact that the Riemannian metric is right-invariant, one obtains

$$
\begin{aligned}
\mathcal{D}_{1} \mathcal{C}(\hat{\mathbf{H}}, \mathbf{C})[\mathbf{U} \hat{\mathbf{H}}] & =\left\langle\operatorname{grad}_{1} \mathcal{C}(\hat{\mathbf{H}}, \mathbf{C}), \mathbf{U} \hat{\mathbf{H}}\right\rangle_{\mathbf{H}} \\
& =\left\langle\left(\operatorname{grad}_{1} \mathcal{C}(\hat{\mathbf{H}}, \mathbf{C})\right) \hat{\mathbf{H}}^{-1} \hat{\mathbf{H}}, \mathbf{U} \hat{\mathbf{H}}\right\rangle_{\mathbf{H}} \\
& =\left\langle\left(\operatorname{grad}_{1} \mathcal{C}(\hat{\mathbf{H}}, \mathbf{C})\right) \hat{\mathbf{H}}^{-1}, \mathbf{U}\right\rangle \\
& =\langle\boldsymbol{\Delta}(\hat{\mathbf{H}}, \mathbf{C}), \mathbf{U}\rangle,
\end{aligned}
$$

with some $\mathbf{U} \in \mathfrak{s l}(3)$. On the other hand, from (8) one has

$$
\begin{aligned}
& \mathcal{D}_{1} \mathcal{C}(\hat{\mathbf{H}}, \mathbf{C})[\mathbf{U} \hat{\mathbf{H}}]=d_{\hat{\mathbf{H}}} \mathcal{C}(\hat{\mathbf{H}}, \mathbf{C})[\mathbf{U} \hat{\mathbf{H}}] \\
& =\sum_{k=1}^{N} \operatorname{tr}\left(\left(\hat{\mathbf{H}}^{-\top} \mathbf{C}_{k} \hat{\mathbf{H}}^{-1}-\dot{\mathbf{C}}_{k}\right) \mathbf{K}_{k}\right. \\
& \left.\quad\left(\left(-\mathbf{U}^{\top} \hat{\mathbf{H}}^{-\top}\right) \mathbf{C}_{k} \hat{\mathbf{H}}^{-1}+\hat{\mathbf{H}}^{-\top} \mathbf{C}_{k}\left(-\hat{\mathbf{H}}^{-1} \mathbf{U}\right)\right)\right) \\
& =-\operatorname{tr}\left(\sum_{k=1}^{N}\left(\mathbf{e}_{k}-\stackrel{\mathbf{C}}{k}_{k}\right) \mathbf{K}_{k}\left(\mathbf{U}^{\top} \mathbf{e}_{k}+\mathbf{e}_{k} \mathbf{U}\right)\right) \\
& =-\operatorname{tr}\left(\sum_{k=1}^{N}\left(\mathbf{e}_{k}\left(\mathbf{e}_{k}-\stackrel{\mathbf{C}}{k}_{k}\right) \mathbf{K}_{k}+\mathbf{e}_{k} \mathbf{K}_{k}\left(\mathbf{e}_{k}-\stackrel{\circ}{\mathbf{C}}_{k}\right)\right) \mathbf{U}^{\top}\right) \\
& =-\left\langle\mathbb{P}\left(\sum_{k=1}^{N} \mathbf{e}_{k}\left(\mathbf{e}_{k}-\stackrel{\mathbf{C}}{k}_{k}\right) \mathbf{K}_{k}+\mathbf{e}_{k} \mathbf{K}_{k}\left(\mathbf{e}_{k}-\stackrel{\mathbf{C}}{k}_{k}\right)\right), \mathbf{U}\right\rangle
\end{aligned}
$$

Then, one directly deduces from (12) and (13) the expression of the innovation term $\boldsymbol{\Delta}(\hat{\mathbf{H}}, \mathbf{C})$ as

$$
\boldsymbol{\Delta}(\hat{\mathbf{H}}, \mathbf{C})=-\mathbb{P}\left(\sum_{k=1}^{N} \mathbf{e}_{k}\left(\mathbf{e}_{k}-\stackrel{\circ}{\mathbf{C}}_{k}\right) \mathbf{K}_{k}+\mathbf{e}_{k} \mathbf{K}_{k}\left(\mathbf{e}_{k}-\stackrel{\circ}{\mathbf{C}}_{k}\right)\right)
$$

One deduces from (14) that $\boldsymbol{\Delta}(\mathbf{E}, \stackrel{\mathbf{C}}{)})=\boldsymbol{\Delta}(\hat{\mathbf{H}}, \mathbf{C})$ and, subsequently, from (7) that 


$$
\dot{\mathbf{E}}=-\mathbb{P}\left(\sum_{k=1}^{N} \mathbf{e}_{k}\left(\mathbf{e}_{k}-\stackrel{\circ}{\mathbf{C}}_{k}\right) \mathbf{K}_{k}+\mathbf{e}_{k} \mathbf{K}_{k}\left(\mathbf{e}_{k}-\stackrel{\circ}{\mathbf{C}}_{k}\right)\right) \mathbf{E}
$$

with $\mathbf{e}_{k}=\mathbf{E}^{-\top} \dot{\mathbf{C}}_{k} \mathbf{E}^{-1}$.

Theorem 1 Consider the autonomous error system (15). Assume that condition (10) is satisfied. Then, the equilibrium $\mathbf{E}=\mathbf{I}$ of System (15) is locally exponentially stable.

The proof is a direct result of [12, Theorem 2]. It remains to determine more explicit conditions under which condition (10) is satisfied, thereby ensuring the validity of Theorem 1.

\section{Observability analysis}

The following lemma provides an explicit sufficient condition for the satisfaction of the algebraic constraint (10) and thus the local observability of the homography.

Lemma 2 (Local observability) Assume that there exist two non-degenerate conics $\stackrel{\mathbf{C}}{1}_{1}, \stackrel{\mathbf{C}}{2}_{2}$ belonging to the set $\mathcal{S}_{C}^{N}$ of all the observed conics such that $\mathbf{M}$, defined by $\mathbf{M} \triangleq \mathbf{C}_{1} \mathbf{C}_{2}^{-1}$, has three distinct non-null eigenvalues. Then, (10) is satisfied and, consequently, the result of Theorem 1 holds.

Proof: One has

$$
\begin{aligned}
\mathrm{d} \rho_{\dot{\mathbf{C}}_{k}}(\mathbf{H})[\mathbf{H U}] & =\mathrm{d}\left(\mathbf{H}^{\top} \stackrel{\circ}{\mathbf{C}}_{k} \mathbf{H}\right)[\mathbf{H U}] \\
& =\mathbf{U}^{\top} \mathbf{H}^{\top} \stackrel{\mathbf{C}}{k}_{k} \mathbf{H}+\mathbf{H}^{\top} \stackrel{\mathbf{C}}{k}_{k} \mathbf{H U},
\end{aligned}
$$

with some $\mathbf{U} \in \mathfrak{s l}(3)$. From (16) one deduces

$$
\operatorname{ker}\left(\mathrm{d} \rho_{\dot{\mathbf{C}}_{k}}(\mathbf{I})\right)=\left\{\mathbf{U} \in \mathfrak{s l}(3) \mid\left(\stackrel{\circ}{\mathbf{C}}_{k} \mathbf{U}\right)^{\top}=-\stackrel{\circ}{\mathbf{C}}_{k} \mathbf{U}\right\} .
$$

Therefore,

$$
\bigcap_{k=1}^{N} \operatorname{ker}\left(\mathrm{d} \rho_{\dot{\mathbf{C}}_{k}}(\mathbf{I})\right)=\left\{\mathbf{U} \in \mathfrak{s l}(3) \mid\left(\stackrel{\mathbf{C}}{k}_{k} \mathbf{U}\right)^{\top}=-\stackrel{\mathbf{C}}{k}_{k} \mathbf{U}, \forall \stackrel{\mathbf{C}}{k}_{k} \in \mathcal{S}_{C}^{N}\right\}
$$

We will determine $\mathbf{U} \in \mathfrak{s l}(3)$ such that $\left(\AA_{k} \mathbf{U}\right)^{\top}=-\stackrel{\mathbf{C}}{k}_{k} \mathbf{U}$, for all $k=1, \cdots, N$. The relation $\left(\dot{\mathbf{C}}_{k} \mathbf{U}\right)^{\top}=-\dot{\mathbf{C}}_{k} \mathbf{U}$ means that $\stackrel{\mathbf{C}}{k}_{k} \mathbf{U}$ is anti-symmetric.

Since $\stackrel{\mathbf{C}}{1}_{1} \mathbf{U}$ and $\stackrel{\circ}{\mathbf{C}}_{2} \mathbf{U}$ are anti-symmetric, there exist $\mathbf{a}_{1}, \mathbf{a}_{2} \in \mathbb{R}^{3}$ such that $\stackrel{\circ}{1}_{1} \mathbf{U}=\mathbf{a}_{1 \times}, \stackrel{\circ}{\mathbf{C}}_{2} \mathbf{U}=\mathbf{a}_{2 \times}$. One deduces

$$
\mathbf{U}=\stackrel{\circ}{\mathbf{C}}_{1}^{-1} \mathbf{a}_{1 \times}=\stackrel{\circ}{\mathbf{C}}_{2}^{-1} \mathbf{a}_{2 \times}
$$

and, subsequently,

$$
\mathbf{a}_{1 \times}=\stackrel{\circ}{\mathbf{C}}_{1} \stackrel{\circ}{\mathbf{C}}_{2}^{-1} \mathbf{a}_{2 \times}=\mathrm{Ma}_{2 \times}
$$

Since $\mathbf{M}$ has three distinct non-null eigenvalues $\lambda_{M 1}, \lambda_{M 2}, \lambda_{M 3}$, it can be diagonalized as $\mathbf{M}=$ $\mathbf{P}_{M} \boldsymbol{\Lambda}_{M} \mathbf{P}_{M}^{-1}$, with some invertible matrix $\mathbf{P}_{M} \in \mathbb{R}^{3 \times 3}$ and $\boldsymbol{\Lambda}_{M}=\operatorname{diag}\left(\lambda_{M 1}, \lambda_{M 1}, \lambda_{M 3}\right)$. Using (18) and the property $\mathbf{A}(\mathbf{a} \times \mathbf{b})=\operatorname{det}(\mathbf{A})\left(\mathbf{A}^{-\top} \mathbf{a} \times \mathbf{A}^{-\top} \mathbf{b}\right)$ for any invertible matrix $\mathbf{A} \in \mathbb{R}^{3 \times 3}$ and $\mathbf{a}, \mathbf{b} \in \mathbb{R}^{3}$, one obtains

$$
\begin{aligned}
0 & =\mathbf{x}^{\top} \mathbf{a}_{1 \times} \mathbf{x}=\mathbf{x}^{\top} \mathbf{P}_{M} \boldsymbol{\Lambda}_{M} \mathbf{P}_{M}^{-1}\left(\mathbf{a}_{2} \times \mathbf{x}\right) \\
& =\operatorname{det}\left(\mathbf{P}_{M}\right)^{-1}\left(\boldsymbol{\Lambda}_{M} \overline{\mathbf{x}}\right)^{\top}\left(\overline{\mathbf{a}}_{2} \times \overline{\mathbf{x}}\right), \forall \mathbf{x} \in \mathbb{R}^{3} .
\end{aligned}
$$

with $\overline{\mathbf{x}} \triangleq \mathbf{P}_{M}^{\top} \mathbf{x}$ and $\overline{\mathbf{a}}_{2} \triangleq \mathbf{P}_{M}^{\top} \mathbf{a}_{2}$. Thus, one deduces

$$
\begin{aligned}
& \left(\lambda_{M 2}-\lambda_{M 1}\right) \bar{a}_{23} \bar{x}_{1} \bar{x}_{2}+\left(\lambda_{M 3}-\lambda_{M 2}\right) \bar{a}_{21} \bar{x}_{2} \bar{x}_{3} \\
& \quad+\left(\lambda_{M 1}-\lambda_{M 3}\right) \bar{a}_{22} \bar{x}_{3} \bar{x}_{1}=0, \forall \bar{x}_{1}, \bar{x}_{2}, \bar{x}_{3} \in \mathbb{R},
\end{aligned}
$$

with $\bar{a}_{21}, \bar{a}_{22}, \bar{a}_{23}$ the 3 components of $\overline{\mathbf{a}}_{2}$. From here, it is straightforward to verify that $\bar{a}_{21}=\bar{a}_{22}=\bar{a}_{23}=0$ (i.e. $\overline{\mathbf{a}}_{2}=\mathbf{0}$ ), which implies that $\mathbf{a}_{2}=\mathbf{P}_{M}^{-\top} \overline{\mathbf{a}}_{2}=\mathbf{0}$. This in turn implies that $\mathbf{U}=\mathbf{0}$ and concludes the proof.

It is noticeable that the homography matrix $\mathbf{H} \in S L(3)$ has 8 degrees of freedom whereas a conic provides 5 degrees of freedom. It is thus expected that 2 conic correspondences would be sufficient to recover $\mathbf{H}$ algebraically. However, this is not always the case. For instance, the correspondences of 2 concentric circles do not determine the homography uniquely since concentric circles are invariant to rotations around their centre. Now, for further discussions, let us assume that $N=2$ (i.e. only 2 conic correspondences) and that $\stackrel{\mathbf{C}}{1}_{1}, \stackrel{\mathbf{C}}{2}_{2}$ and $\mathbf{M}\left(=\dot{\mathbf{C}}_{1} \mathbf{C}_{2}^{-1}\right)$ are non-degenerate. We now investigate the cases where $\mathbf{M}$ has a multiple eigenvalue so that the sufficient observability condition of Lemma 2 is violated.

- Case of M having a triple eigenvalue: This case implies that $\mathbf{M}=\mathbf{I}$ using that fact that $\operatorname{det}(\mathbf{M})=$ $\operatorname{det}\left(\stackrel{\circ}{\mathbf{C}}_{1}\right) \operatorname{det}\left(\stackrel{\circ}{\mathbf{C}}_{2}\right)^{-1}=1$. Thus, $\stackrel{\circ}{\mathbf{C}}_{1}=\stackrel{\circ}{\mathbf{C}}_{2}$, meaning that the two conics coincide (i.e. only one conic correspondence is available). In this case, $\mathbf{H}$ is clearly unobservable.

- Case of $\mathbf{M}$ having a double eigenvalue (i.e. $\lambda_{M 1}=$ $\left.\lambda_{M 2} \neq \lambda_{M 3}\right)$ and diagonalizable as $\mathbf{M}=\mathbf{P}_{M} \boldsymbol{\Lambda}_{M} \mathbf{P}_{M}^{-1}$ with some invertible matrix $\mathbf{P}_{M} \in \mathbb{R}^{3 \times 3}$ and $\boldsymbol{\Lambda}_{M}=$ $\operatorname{diag}\left(\lambda_{M 1}, \lambda_{M 1}, \lambda_{M 3}\right)$ :

When does this case occur? Define a "virtual homography" $\mathbf{H}_{M} \triangleq \operatorname{det}\left(\mathbf{P}_{M}\right)^{\frac{1}{3}} \mathbf{P}_{M}^{-\top} \in S L(3)$, and $\mathbf{C}_{1 M} \triangleq \mathbf{H}_{M}^{\top} \mathbf{C}_{1} \mathbf{H}_{M}$, $\mathbf{C}_{2 M} \triangleq \mathbf{H}_{M}^{\top} \stackrel{\mathbf{C}}{2}_{2} \mathbf{H}_{M}$, representing the projective transformation by $\mathbf{H}_{M}$ of $\dot{\mathbf{C}}_{1}, \dot{\mathbf{C}}_{2}$, respectively. One deduces from $\mathbf{M}=\mathbf{P}_{M} \boldsymbol{\Lambda}_{M} \mathbf{P}_{M}^{-1}$ that

$$
\begin{aligned}
& \mathbf{C}_{1 M} \mathbf{C}_{2 M}^{-1}=\boldsymbol{\Lambda}_{M}=\lambda_{1 M} \mathbf{I}+\left(\lambda_{3 M}-\lambda_{1 M}\right) \mathbf{e}_{3} \mathbf{e}_{3}^{\top} \\
\Rightarrow & \mathbf{C}_{1 M}=\lambda_{1 M} \mathbf{C}_{2 M}+\left(\lambda_{3 M}-\lambda_{1 M}\right) \mathbf{e}_{3} \mathbf{e}_{3}^{\top} \mathbf{C}_{2 M}
\end{aligned}
$$

Since $\mathbf{C}_{1 M}$ and $\mathbf{C}_{2 M}$ are symmetric and $\lambda_{3 M} \neq \lambda_{1 M}$, one deduces that $\mathbf{e}_{3} \mathbf{e}_{3}^{\top} \mathbf{C}_{2 M}=\mathbf{C}_{2 M} \mathbf{e}_{3} \mathbf{e}_{3}^{\top}$, from which one obtains the following form of $\mathbf{C}_{2 M}$

$$
\mathbf{C}_{2 M}=\left[\begin{array}{ccc}
a_{2 M} & b_{2 M} & 0 \\
b_{2 M} & c_{2 M} & 0 \\
0 & 0 & f_{2 M}
\end{array}\right]
$$

and, thus,

$$
\mathbf{C}_{1 M}=\lambda_{1 M}\left[\begin{array}{ccc}
a_{2 M} & b_{2 M} & 0 \\
b_{2 M} & c_{2 M} & 0 \\
0 & 0 & \frac{1}{\lambda_{1 M}^{3}} f_{2 M}
\end{array}\right]
$$

when using the relation $\operatorname{det}(\mathbf{M})=\lambda_{1 M}^{2} \lambda_{3 M}=1$. From (21) and (22), it is straightforward to deduce that $\mathbf{C}_{1 M}$ and $\mathbf{C}_{2 M}$ (resp. $\dot{\mathbf{C}}_{1}$ and $\dot{\mathbf{C}}_{2}$ ) are concentric, of the same type, and scale of each other. Since they are non-degenerate, they cannot be parabolas and, thus, must be both ellipses or hyperbolas.

Now, proceeding analogously to the proof of Lemma 2 one deduces (see Eq. (19))

$$
\left(\boldsymbol{\Lambda}_{M} \overline{\mathbf{x}}\right)^{\top}\left(\overline{\mathbf{a}}_{2} \times \overline{\mathbf{x}}\right)=0, \quad\left(\boldsymbol{\Lambda}_{M}^{-1} \overline{\mathbf{x}}\right)^{\top}\left(\overline{\mathbf{a}}_{1} \times \overline{\mathbf{x}}\right)=0, \quad \forall \overline{\mathbf{x}} \in \mathbb{R}^{3},
$$

with $\overline{\mathbf{a}}_{1} \triangleq \mathbf{P}_{M}^{\top} \mathbf{a}_{1}, \overline{\mathbf{a}}_{2} \triangleq \mathbf{P}_{M}^{\top} \mathbf{a}_{2}$. Since $\lambda_{M 1}=\lambda_{M 2} \neq \lambda_{M 3}$, one can only deduce that $\bar{a}_{11}=\bar{a}_{12}=\bar{a}_{21}=\bar{a}_{22}=0$. Thus,

$$
\begin{aligned}
& \mathbf{a}_{1}=\mathbf{P}_{M}^{-\top} \overline{\mathbf{a}}_{1}=\bar{a}_{13} \mathbf{P}_{M}^{-\top} \mathbf{e}_{3}, \\
& \mathbf{a}_{2}=\mathbf{P}_{M}^{-\top} \overline{\mathbf{a}}_{2}=\bar{a}_{23} \mathbf{P}_{M}^{-\top} \mathbf{e}_{3} .
\end{aligned}
$$




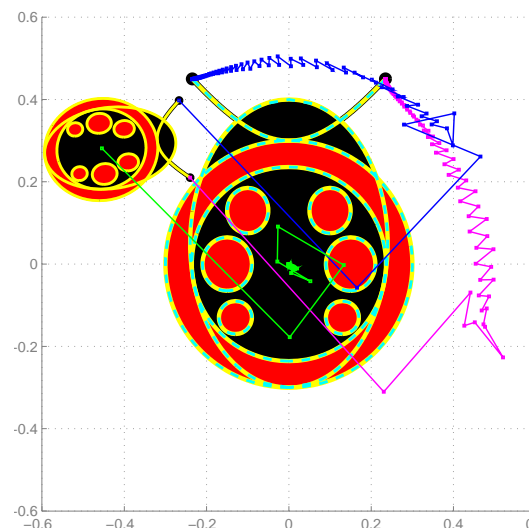

(A) Use of all 10 conics

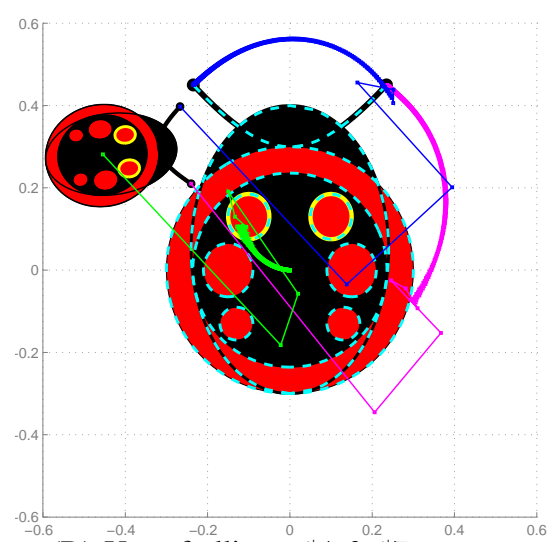

(D) Use of ellipses $\sharp 4 \& \sharp 7$

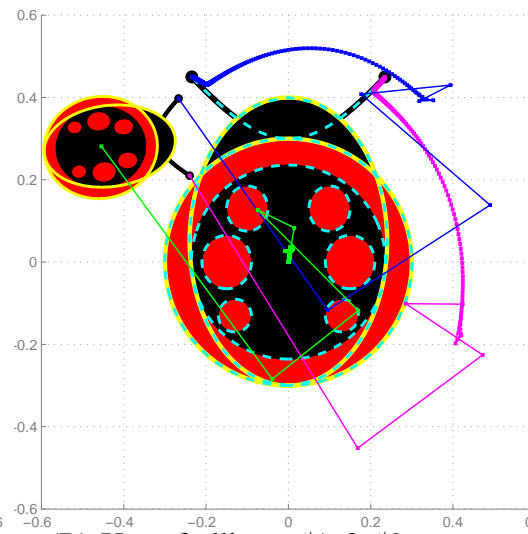

(B) Use of ellipses $\sharp 1 \& \stackrel{0.2}{-0.2} \sharp 2$

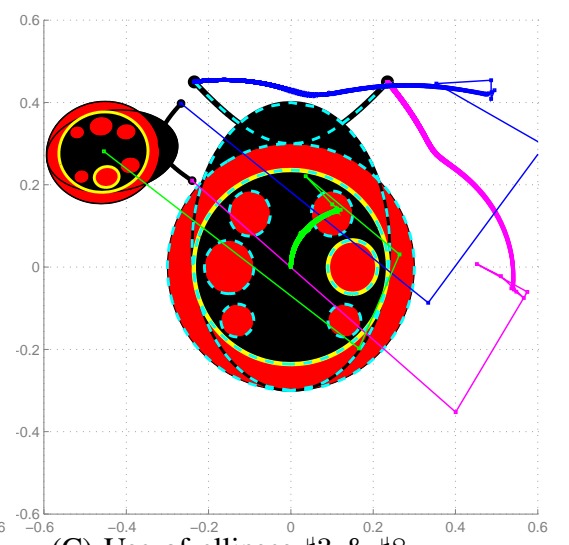

(C) Use of ellipses $\sharp 3 \& \stackrel{0.2}{\text { \& }} \sharp 8$

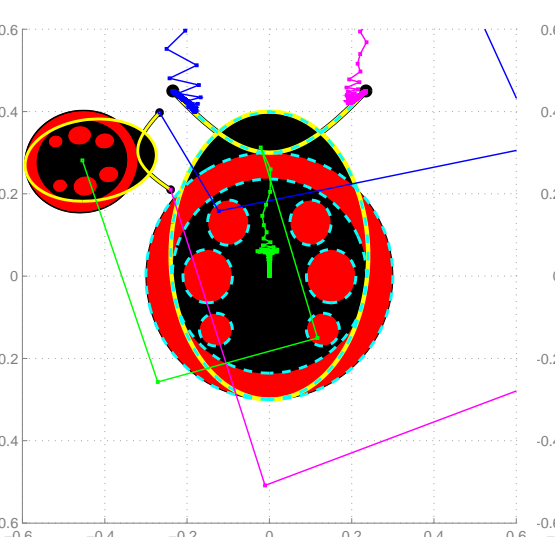

(E) Use of ellipse $\sharp 1$ \& hyperbola $\sharp 10$

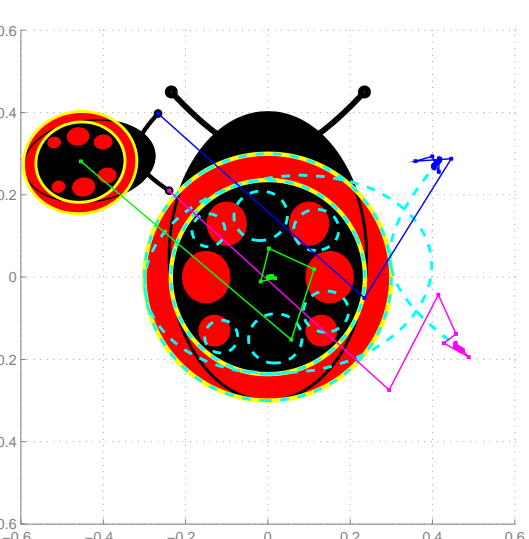

(F) Use of concentric circles $\sharp 2^{0.4} \& \sharp 3^{0.6}$

Fig. 1: Simulation results illustrating the performance of the proposed algorithm by using 6 combinations of conic correspondences: 5 observable cases $(A-E)$ and 1 unobservable case $(F)$. Both reference and current views of the "ladybird" are put in the same figures; warped current conics using the final estimated homography are presented by cyan and dashed conics; the evolutions of the iterated homography estimates $\left\{\hat{\mathbf{H}}_{k}\right\}$ are presented by 3 curves (green, blue, magenta) obtained from the projective transformation of 3 points of the "ladybird" by these homographies.

Therefore, in contrast with Lemma 2, two conic correspondences in this case are not sufficient to deduce $\mathbf{a}_{1}=\mathbf{a}_{2}=0$ (i.e. $\mathbf{U}=\mathbf{0}$ ), meaning that neither (10) nor the observability of $\mathbf{H}$ is guaranteed.

\section{Simulation Results}

In this section simulation results are presented to illustrate the performance of the proposed observer (i.e. (6)+(14)) applied to the estimation of the homography relating two views of a planar scene (static case, i.e. $\mathbf{U} \equiv \mathbf{0}$ ).

A discrete version of the proposed observer is implemented using the accelerated line search (ALS) algorithm on manifolds proposed in [1, Page 63]. In more detail, by adapting that ALS algorithm to our observer and by setting $\mathbf{U}$ in (6) equal to zero, the update formula at each iteration step is given by

$$
\hat{\mathbf{H}}_{k+1}=e^{-\boldsymbol{\Delta}_{k} t_{k}} \hat{\mathbf{H}}_{k}
$$

with $\boldsymbol{\Delta}_{k}$ calculated according to (14) using $\hat{\mathbf{H}}_{k}$ in the replacement of $\hat{\mathbf{H}}$, and $t_{k}$ the step length obtained from

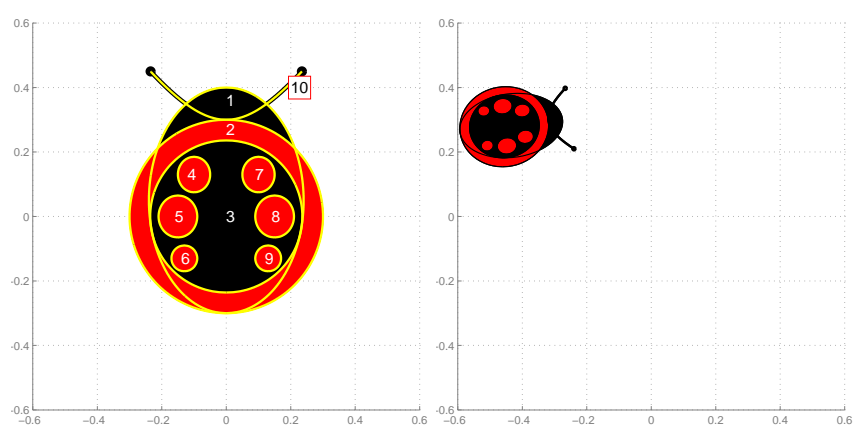

(A) Reference view

(B) Current view

Fig. 2: Left: the simulation scene that we call "ladybird" is composed of 10 conics $(9$ ellipses or circles and 1 hyperbola), where conics $\sharp 2$ and $\sharp 3$ are concentric circles and conic $\sharp 10$ is a hyperbola. Right: the current view of the same scene by application of a homography corresponding to a large motion in both rotation and translation. 


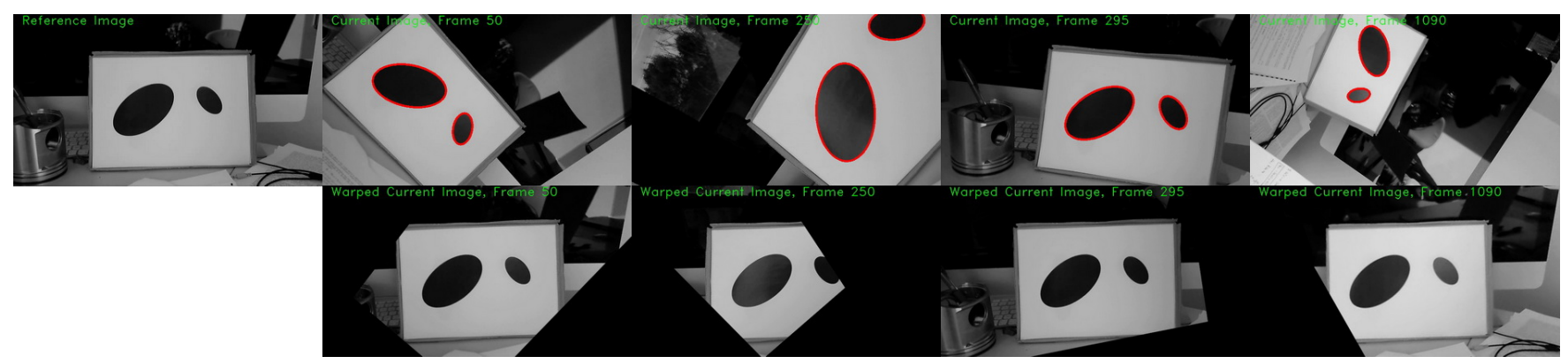

Fig. 3: Experiment 1 (Observable case): Homography estimation using the correspondences of two ellipses (red color) with distinct centres. Reference image on top left; 4 current image frames (50, 250, 295, 1090) and their associated warped images using the homography estimates.

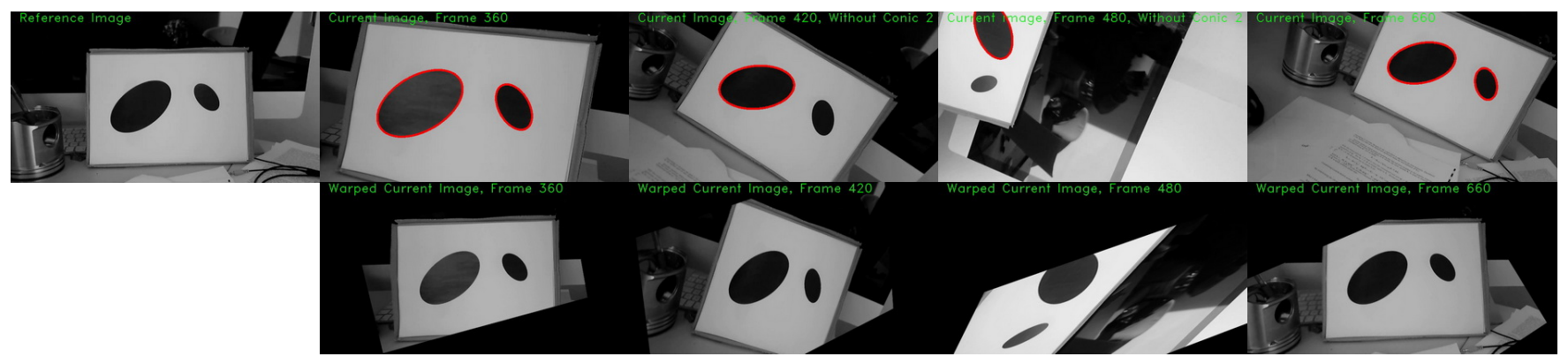

Fig. 4: Experiment 1 (Unobservable case): One of the 2 ellipse correspondences is intentionally removed from homography estimation from second 25 to second 35. During that period only the observed ellipse is stabilized in the warped images but not the whole scene. The correct homography estimates are quickly recovered when both ellipse correspondences are again available (after second 35 ).

Armijo's backtracking procedure (see [1, Page 62]) that relies on the following Armijo-like condition

$$
\mathcal{C}\left(\hat{\mathbf{H}}_{k}, \mathbf{C}\right)-\mathcal{C}\left(\hat{\mathbf{H}}_{k+1}, \mathbf{C}\right) \geq t_{k}^{A} \sigma\left\|\boldsymbol{\Delta}_{k}\right\|_{F}^{2}
$$

with $t_{k}^{A}\left(=\beta^{m} \bar{\alpha}\right)$ the Armijo step size [1, Definition 4.2.2] for the given $\bar{\alpha}, \beta, \sigma, \boldsymbol{\Delta}_{k}$. The chosen parameters are $\bar{\alpha}=$ $0.05, \sigma=0.25, \beta=0.75$. Finally, all gain matrices $\mathbf{K}_{k}$ involved in the expression (14) of $\boldsymbol{\Delta}_{k}$ are taken as diag $(1,1,2)$.

The reference and current views of the simulated "ladybird" planar scene composed of 10 conics ( 9 ellipses or circles and 1 hyperbola) are depicted in Fig. 2. A large number of conics is deliberately chosen so as to show that the proposed algorithm can easily handle such a situation. Six simulation tests have been performed using the correspondences of:

1) all 10 conics [Fig. 1(A)];

2) ellipses $\sharp 1$ and $\sharp 2$ [Fig. 1(B)];

3) ellipses $\sharp 3$ and $\sharp 8$ [Fig. 1(C)];

4) ellipses $\sharp 4$ and $\sharp 7$ [Fig. 1(D)];

5) ellipse $\sharp 1$ and hyperbola $\sharp 10$ [Fig. 1(E)];

6) concentric circles $\sharp 2$ and $\sharp 3$ [Fig. 1(F)];

The results are presented in Figs. 1(A-F) where the 10 warped conics, plotted in cyan color and dashed curves, are obtained from projective transformation of the 10 current conics by the estimated homography matrices according to
(5). The superposition of the warped conics and the reference ones is an indicator of successful homography estimation for the 5 first cases (see Figs. 1(A)-1(E)). On the contrary, the use of correspondences of two concentric circles $\sharp 2$ and $\sharp 3$ (Case 6) no longer provides a correct homography estimate as shown in Fig. 1(F) where only the used conics $\sharp 2$ and $\sharp 3$ are correctly warped. This illustrates the unobservability issue discussed in Section III-C.

\section{EXPERIMENTAL RESULTS - IMAGE STABILIZATION}

In this section an application of the proposed algorithm to image stabilization is presented. The experimentations have been performed with a classical webcam that provides 20 images per second with a resolution of $752 \times 416$ pixels. The estimated camera parameters are $(792,768)$ pixels for the focal length and $(375.5,207.5)$ pixels for the principle point. Code has been implemented in $\mathrm{C}++$ with ViSP library [14]. The vpMeEllipse Class is used for real-time tracking of selected ellipses and, subsequently, their associated equation. After the step of feature tracking, the ASL version of the proposed observer is iterated with the maximum number of iteration equal to 2500 per image frame. The computational effort for this last step is negligible compared to the previous image processing step and does not affect the real-time capability of the proposed approach. Since the group velocity $\mathrm{U}$ is not measured, it is simply set to zero. This in turn allows 


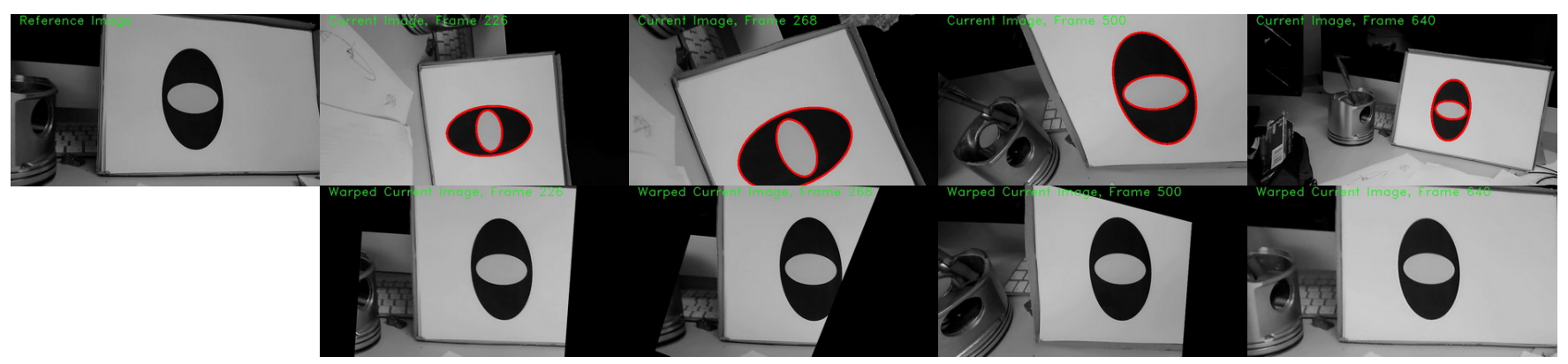

Fig. 5: Experiment 2 (Observable case): Homography estimation using the correspondences of two ellipses (red color) with same centre. Reference image on top left; 4 current image frames $(226,268,500,640)$ and their associated warped images using the homography estimates.

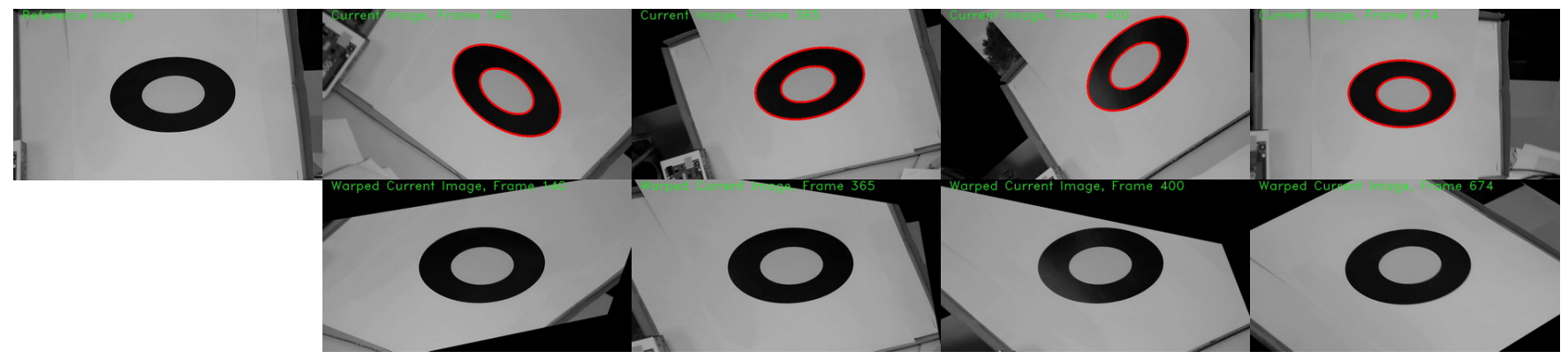

Fig. 6: Experiment 3 (Unobservable case): Homography estimation using the correspondences of two equivalent ellipses (red color) with same centre, same form, same orientation but different scales. Reference image on top left; 4 current image frames $(140,365,400,674)$ and their associated warped images using the homography estimates. Only the observed ellipses are stabilized in the warped images but not the whole scene.

us to validate the robustness of the proposed approach with respect to the unavailability of such measurements.

Three experiments have been carried out using the correspondences of:

1) 2 ellipses with distinct centres (Experiment 1);

2) 2 ellipses with same centre (Experiment 2);

3) 2 equivalent ellipses with same centre, same form, same orientation but different scales (Experiment 3).

Some screenshots of these experiments are depicted in Figs. 3-6. A video of full experimental results is available at

$$
\text { http://goo.gl/8wFGb5 }
$$

showing good and robust performance of the proposed approach. It can be observed that the reference image is successfully stabilized by the warped current images when the sufficient observability condition in Lemma 2 is satisfied (when using two conic correspondences) as in Experiment 1 (Fig 3) and Experiment 2 (Fig 5). On the other hand, when one of the two ellipse correspondences is intentionally removed from homography estimation from second 25 to second 35 in Experiment 1, the homography estimates become erroneous during that period of unobservability (Fig. 4). However, the proposed algorithm still continues to operate and exploit the remaining ellipse correspondence for homography estimation (the used ellipse is still stabilized in the warped image) and quickly recovers correct homogra- phy estimates when two ellipse correspondences are again available (after second 35). This illustrates the robustness of our algorithm. As for Experiment 3 that corresponds to an unobservable case discussed in Section III-C where the matrix $\mathbf{M}=\dot{\mathbf{C}}_{1} \stackrel{\circ}{\mathbf{C}}_{2}^{-1}$ has a double eigenvalue, it can be observed that, similarly to Case 6 in the simulation section IV, only the two observed conics are stabilized in the warped current images but not the whole scene (Fig. 6). This again allows us to confirm the observability analysis given in Section III-C.

\section{CONCLUSIONS}

A nonlinear observer designed on the Special Linear group $S L(3)$ for the estimation of a sequence of homographies has been proposed. It extends our prior works on this topic by exploiting conics as image features. The proposed observer directly incorporates conic correspondences from an image sequence without requiring explicit computation of individual homographies between images. The observability issue associated with the considered image features has been carefully addressed. While only local exponential stability of the observer can be proved with the kind of cost function considered, simulation and experimental results have been provided as a complement to the theoretical approach in order to demonstrate a large domain of stability. 
Acknowledgement: This research was supported by the ANR ASTRID SCAR project (ANR-12-ASTR-0033), the ROBOTEX project (ANR-10-EQPX-44) and the Australian Research Council through the "Australian Centre of Excellence for Robotic Vision" (CE140100016).

\section{REFERENCES}

[1] P-A. Absil, R. Mahony, and R. Sepulchre. Optimization algorithms on matrix manifolds. Princeton University Press, 2009.

[2] A. Agarwal, C.V. Jawahar, and P.J. Narayanan. A survey of planar homography estimation techniques. Centre for Visual Information Technology, Tech. Rep. IIIT/TR/2005/12, 2005.

[3] C. Conomis. Conics-based homography estimation from invariant points and pole-polar relationships. In IEEE Third Int. Symp. on $3 D$ Data Processing, Visualization, and Transmission, pp. 908-915, 2006.

[4] T. Hamel, R. Mahony, J. Trumpf, P. Morin, and M.-D. Hua. Homography estimation on the Special Linear group based on direct point correspondence. In IEEE Conf. on Decision and Control (CDC), pp. 7902-7908, 2011

[5] R. Hartley and A. Zisserman. Multiple View Geomerty in Computer Vision. Cambridge University Press, second edition, 2003.

[6] M.-D. Hua, J. Trumpf, T. Hamel, R. Mahony, and P. Morin. Point and line feature-based observer design on $S L(3)$ for Homography estimation and its application to image stabilization. To appear in IEEE Int. Conf. on Robotics and Automation (ICRA), 2017.

[7] P.K. Jain. Homography estimation from planar contours. In IEEE Third Int. Symp. on 3D Data Processing, Visualization, and Transmission, pp. 877-884, 2006.

[8] J.Y. Kaminski and A. Shashua. Multiple view geometry of general algebraic curves. Int. J. of Computer Vision, 56(3):195-219, 2004.

[9] J. Kannala, M. Salo, and J. Heikkila. Algorithms for Computing a Planar Homography from Conics in Correspondence. In $B M V C$, pp. 77-86, 2006.

[10] Y. Ma, S. Soatto, J. Kosecka, and S.S. Sastry. An Invitation to 3-D Vision: From Images to Geometric Models. Springer Verlag, 2003.

[11] R. Mahony, T. Hamel, P. Morin, and E. Malis. Nonlinear complementary filters on the special linear group. Int. J. of Control, 85(10): 1557-1573, 2012.

[12] R. Mahony, J. Trumpf, and T. Hamel. Observers for Kinematic Systems with Symmetry. In 9th IFAC Symp. on Nonlinear Control Systems, pp. 617-633, 2013.

[13] E. Malis, T. Hamel, R. Mahony, and P. Morin. Dynamic estimation of homography transformations on the special linear group for visual servo control. In IEEE Int. Conf. on Robotics and Automation (ICRA), pp. 1498-1503, 2009.

[14] E. Marchand, F. Spindler, and F. Chaumette. ViSP for visual servoing: a generic software platform with a wide class of robot control skills. IEEE Robotics and Automation Magazine, 12(4):40-52, 2005.

[15] A. Sugimoto. A linear algorithm for computing the homography from conics in correspondence. J. of Mathematical Imaging and Vision, 13(2): 115-130, 2000. 